\title{
BMC Obesity reviewer acknowledgement 2014
}

\section{Clare Partridge}

\section{Contributing reviewers}

The editors of BMC Obesity would like to thank all our reviewers who have contributed to the journal in Volume 1 (2014).

\begin{tabular}{|c|c|c|}
\hline $\begin{array}{l}\text { Sean Adams } \\
\text { USA }\end{array}$ & $\begin{array}{l}\text { George Chrousos } \\
\text { Greece }\end{array}$ & $\begin{array}{l}\text { Folke Hammarqvist } \\
\text { Sweden }\end{array}$ \\
\hline $\begin{array}{l}\text { Raghu Adya } \\
\text { UK }\end{array}$ & $\begin{array}{l}\text { Anna Ciao } \\
\text { USA }\end{array}$ & $\begin{array}{l}\text { Thang Han } \\
\text { UK }\end{array}$ \\
\hline $\begin{array}{l}\text { Carlos Alvarez-Dardet } \\
\text { Spain }\end{array}$ & $\begin{array}{l}\text { Eva Conceicao } \\
\text { Portugal }\end{array}$ & $\begin{array}{l}\text { Flo Harrison } \\
\text { UK }\end{array}$ \\
\hline $\begin{array}{l}\text { Raghupathy Anchala } \\
\text { India }\end{array}$ & $\begin{array}{l}\text { Molly Conroy } \\
\text { USA }\end{array}$ & $\begin{array}{l}\text { Lana Hebden } \\
\text { Australia }\end{array}$ \\
\hline $\begin{array}{l}\text { Shervin Assari } \\
\text { USA }\end{array}$ & $\begin{array}{l}\text { Russell De Souza } \\
\text { Canada }\end{array}$ & $\begin{array}{l}\text { Setia Hermawati } \\
\text { UK }\end{array}$ \\
\hline $\begin{array}{l}\text { Navoda Atapattu } \\
\text { Sri Lanka }\end{array}$ & $\begin{array}{l}\text { Jeffrey Deiuliis } \\
\text { USA }\end{array}$ & $\begin{array}{l}\text { Natasha Howard } \\
\text { Australia }\end{array}$ \\
\hline $\begin{array}{l}\text { Kathryn Backholer } \\
\text { Australia }\end{array}$ & $\begin{array}{l}\text { Hélène Delisle } \\
\text { Canada }\end{array}$ & $\begin{array}{l}\text { Terry Huang } \\
\text { USA }\end{array}$ \\
\hline $\begin{array}{l}\text { Katherine Bauer } \\
\text { USA }\end{array}$ & $\begin{array}{l}\text { Flávio Demarco } \\
\text { Brazil }\end{array}$ & $\begin{array}{l}\text { Melinda Hutchesson } \\
\text { Australia }\end{array}$ \\
\hline $\begin{array}{l}\text { Grzegorz Bazylak } \\
\text { Poland }\end{array}$ & $\begin{array}{l}\text { Jacopo Desiderio } \\
\text { Italy }\end{array}$ & $\begin{array}{l}\text { Kevin Hwang } \\
\text { USA }\end{array}$ \\
\hline $\begin{array}{l}\text { Colin Bell } \\
\text { Australia }\end{array}$ & $\begin{array}{l}\text { Rosemary Duda } \\
\text { USA }\end{array}$ & $\begin{array}{l}\text { Judy Jou } \\
\text { USA }\end{array}$ \\
\hline $\begin{array}{l}\text { Rhonda Belue } \\
\text { USA }\end{array}$ & $\begin{array}{l}\text { Jozef Dulak } \\
\text { Poland }\end{array}$ & $\begin{array}{l}\text { Gregor Jurak } \\
\text { Slovenia }\end{array}$ \\
\hline $\begin{array}{l}\text { Farryl Bertmann } \\
\text { USA }\end{array}$ & $\begin{array}{l}\text { Mary Forhan } \\
\text { Canada }\end{array}$ & $\begin{array}{l}\text { Masaharu Kagawa } \\
\text { Japan }\end{array}$ \\
\hline $\begin{array}{l}\text { Ken Blemings } \\
\text { USA }\end{array}$ & $\begin{array}{l}\text { Rosanne Freak-Poli } \\
\text { Australia }\end{array}$ & $\begin{array}{l}\text { Juliana Kain } \\
\text { Chile }\end{array}$ \\
\hline $\begin{array}{l}\text { Susann Bluher } \\
\text { Germany }\end{array}$ & $\begin{array}{l}\text { Ismael Freitas } \\
\text { Brazil }\end{array}$ & $\begin{array}{l}\text { Gregory Kaltsas } \\
\text { Greece }\end{array}$ \\
\hline $\begin{array}{l}\text { Maria Bryant } \\
\text { UK }\end{array}$ & $\begin{array}{l}\text { Barry Garst } \\
\text { USA }\end{array}$ & $\begin{array}{l}\text { Rebecca Kanter } \\
\text { USA }\end{array}$ \\
\hline $\begin{array}{l}\text { Mansi Chopra } \\
\text { India }\end{array}$ & $\begin{array}{l}\text { Sanne Gerards } \\
\text { Netherlands }\end{array}$ & $\begin{array}{l}\text { Olga Khavjou } \\
\text { USA }\end{array}$ \\
\hline
\end{tabular}

BioMed Central, Floor 6, 236 Gray's Inn Road, London WC1X 8HB, United Kingdom 
Isurujith Kongalaliyanage

Sri Lanka

Bharati Kulkarni

India

Amy Luke

USA

Gian Mauro Manzoni

Italy

Anne Martin

UK

Katherine McCullough

UK

Daniel Mitchell

UK

Thomas Modéer

Sweden

Victor Mogre

Ghana

Paulina Nowicka

Sweden

Christine Olson

USA

Carsten Otto

Germany

Miranda Pallan

UK
Ashan Pathirana

Sri Lanka

Christine Peat

USA

Anna Peeters

Australia

Ralph Peterli

Switzerland

Antonio Prista

Mozambique

Kumari Rathnayake

Sri Lanka

Francisco Rios

UK

Anne Elizabeth Rogers

UK

Stefan Ryter

USA

Ian Sinha

UK

Amanda Staiano

USA

Steven Street

Australia

Michel Suter

Switzerland
Kurt Svardsudd

Sweden

George Tharakan

UK

Kimberly Truesdale

USA

Adam Tsai

USA

Indu Waidyatilaka

Sri Lanka

Carol Wham

New Zealand

Brandy Wicklow

Canada

Pujitha Wickramasinghe

Sri Lanka

Andrew James Williams

UK

Emmanuel Williams

Ghana

Jyh Eiin Wong

Malaysia

Lin Yang

USA

Ouliana Ziouzenkova

USA 